\title{
Interference-Aware Relay Selection Scheme for Two-Hop Relay Networks With Multiple Source-Destination Pairs
}

\author{
Sheng Zhou, Member, IEEE, Jie Xu, Student Member, IEEE, and Zhisheng Niu, Fellow, IEEE
}

\begin{abstract}
An interference-aware relay selection scheme is proposed for two-hop relay networks with multiple sourcedestination (S-D) pairs, each of which is assisted by a relay selected from the candidates. We address the interference from relays to their nonintended destinations, and the interference level at any destination is jointly affected by relay selections of all $S-D$ pairs. Upper and lower bounds of the two-hop transmission rate are derived, and the joint impact of the interference from multiple relays is decoupled to each $S-D$ pair. Based on these bounds, an interference-aware relay selection metric for each $\mathrm{S}-\mathrm{D}$ pair is proposed, which reflects both the relay-assisted two-hop rate and the induced interference to other destinations. The decoupled metric enables us to formulate the relay selection as a weighted bipartite matching problem, with the $S-D$ pairs and the candidate relays as the two sides. Both the centralized scheme based on the Hungarian algorithm and the distributed scheme based on matching games are presented. Simulation results show that the distributed scheme performs nearly the same as the centralized scheme, and both schemes substantially outperform the conventional min-max relay selection scheme in terms of network sum rate.
\end{abstract}

Index Terms-Bipartite matching, collaborative relay, interference limited system, relay selection.

\section{INTRODUCTION}

A S AN EMERGING technology for future wireless networks, cooperative transmission [1], [2] has been extensively studied recently and has been also adopted in Long-Term Evolution Advanced (LTE-A) systems [3]. Due to the broadcast nature of the wireless medium, a relay can hear the signal of a transmitter in its detection region and forward it to the destination; thus, cooperative diversity is exploited. However, active relays can cause additional interference to neighboring links

Manuscript received March 30, 2012; revised September 24, 2012 and December 19, 2012; accepted December 21, 2012. Date of publication January 9, 2013; date of current version June 12, 2013. This work was supported in part by the National Basic Research Program of China under Grant 2012CB316001, by the Natural Science Foundation of China under Grant 60925002 and Grant 61021001, and by Hitachi Ltd. This paper was presented in part at the 15th Asia-Pacific Conference on Communications, Shanghai, China, October 8-10, 2009. The review of this paper was coordinated by Dr. H. Jiang.

S. Zhou and Z. Niu are with Tsinghua National Laboratory for Information Science and Technology, Department of Electronic Engineering, Tsinghua University, Beijing 100084, China (e-mail: sheng.zhou@tsinghua.edu.cn; niuzhs@tsinghua.edu.cn).

J. Xu was with Tsinghua National Laboratory for Information Science and Technology, Department of Electronic Engineering, Tsinghua University, Beijing 100084, China. He is currently with Department of Electrical Engineering, University of California, Los Angeles, Los Angeles, CA 90095-1594 USA (e-mail: jiexu@ucla.edu).

Color versions of one or more of the figures in this paper are available online at http://ieeexplore.ieee.org.

Digital Object Identifier 10.1109/TVT.2013.2238266
[4]. For example, in relay-assisted cellular networks, relays are used to improve the performance of edge users, but at the same time, they potentially cause interference to neighboring cells. In ad hoc networks, if idle nodes serve as relays, they may cause severe interference to other concurrent transmissions within its interference region [6]. Therefore, interference must be carefully treated for collaborative relays. Relay selection, as one of the fundamental design issues, is the focus of this paper.

There has been much work on relay selection for isolated topologies, in which one or more selected relays assist one source-destination (S-D) pair [7]. In [8], it is shown that selecting one "best" relay achieves the same diversity gain as distributed space-time codes [1]. Two relay selection metrics, "max-min" and "harmonic mean" are proposed. In [9], a scaled version of the harmonic mean of the source-relay and relay-destination channels are used as the selection metric, and the spectral efficiency is increased. Sadek et al. [10] consider the uplink of a single cell with multiple users, and users select relays from their nearest neighbors toward the access point. Game-theoretical approaches are also exploited for relay selection [11]-[14], particularly for distributed implementations. However, none of the above considers interference among relay-assisted transmissions.

Large-scale networks often comprise multiple concurrent transmissions that cannot be perfectly isolated; thus, interference causes performance degradation. This impact becomes even more severe and complicated due to the interference elevated by transmitting relays [4]. The effect of multiuser interference is analyzed with the relay selection in [15], where an extension to conventional max-min selection is proposed, taking the interference from sources to relays into consideration. However, interference from relays to destinations is not dealt with. In [16], both the interference on the relays and that on destinations are analyzed. Nevertheless, the relay selection scheme is purely local, ignoring the impact of interference to other destinations. In [17], Huang et al. use prices to reflect the sensitivity of nodes to the interference level and adjust their power to maximize the profit. A distributed relay selection and spectrum-allocation scheme based on a noncooperative game is proposed in [18], with a conservative interference model assuming that all sources and active relays cause interference on both hops. To summarize, very few studies design relay selection schemes jointly with the consideration of the induced interference from relays.

Therefore, in this paper, we propose an interference-aware relay selection scheme, addressing the potential interference 
elevated from relays. Our first challenge is to characterize the interference from relays. On one hand, the rate of a relay-todestination link depends on multiple interfering relays. On the other hand, an active relay also interferes multiple destinations. Therefore, the impact of the interference couples with the relay selections of multiple S-D pairs. We derive upper and lower bounds of the relay-to-destination link rate, and show that the rate can be written in a linear combination of interference items, only requiring local channel state information (CSI). Then, a decoupled metric for relay selection is derived, which consists of two parts: the conventional max-min metric, with a cost term reflecting the interference induced by the relay. To maximize the sum rate of the network, we model the relay selection as weighted bipartite matching with the S-D pairs and the relays as the two sides, and the proposed metric is the matching weight. We further design centralized and distributed relay selection algorithms based on the Hungarian algorithm and the stable marriage problem [23] from matching game theory [20], respectively. Through extensive simulations, the performance gain of the proposed relay selection scheme over conventional max-min scheme is verified.

The rest of this paper is organized as follows. A system model is introduced in Section II. Section III presents the problem formulation. In Section IV, the impact of multiple interfering relays is decoupled into a linear combination form. Section V derives the relay selection metric. Both centralized and distributed relay selection algorithms are proposed in Section VI. Simulations are provided in Section VII, after which, this paper is concluded in Section VIII.

\section{SYSTEM MODEL}

We consider a wireless network with multiple concurrent S-D transmission pairs. Each S-D pair, which is denoted by $\left(s_{i}, d_{i}\right)$, including source $s_{i}$ and destination $d_{i}$, together with their selected relay, form a transmission cluster. For different network architectures, the cluster has different forms. For example, in relay-assisted cellular networks, a cluster refers to a cell with several relay stations. For unplanned ad hoc networks, the cluster depends on the location of a S-D pair and the idle nodes around them. As shown in Fig. 1, $N$ busy S-D pairs form the source set $\mathcal{S}=\left\{s_{1}, s_{2}, \ldots, s_{N}\right\}$ and the corresponding destination set $\mathcal{D}=\left\{d_{1}, d_{2}, \ldots, d_{N}\right\}$. The set of all S-D pairs is denoted as $\mathcal{P}=\left\{\left(s_{1}, d_{1}\right),\left(s_{1}, d_{2}\right), \ldots,\left(s_{N}, d_{N}\right)\right\}$. The $M$ candidate relays form the set $\mathcal{R}=\left\{r_{1}, r_{2}, \ldots, r_{M}\right\}$. We denote the selected relay for $\mathrm{S}-\mathrm{D}$ pair $\left(s_{i}, d_{i}\right)$ as $k_{i} \in \mathcal{R}$. To focus our work on the relaying process, it is assumed that a direct link from $s_{i}$ to $d_{i}$ is not available (i.e., the source-to-destination channel is with high path loss) and that communication can only be established via two-hop relaying. The reason for making this assumption is that this paper focuses on the interference from relays when making relay selection decisions, whereas the direct link is not affected by relay selections. Moreover, in real systems such as LTE-A, most relays use two-hop transmission without the direct link [3]. In each cluster, two orthogonal channels (time slots or frequency bands, etc.) are available, i.e., source $s_{i}$ transmits, e.g., on channel 1 , and relay $k_{i}$ transmits on channel 2. Although the transmissions of source $s_{i}$ and relay

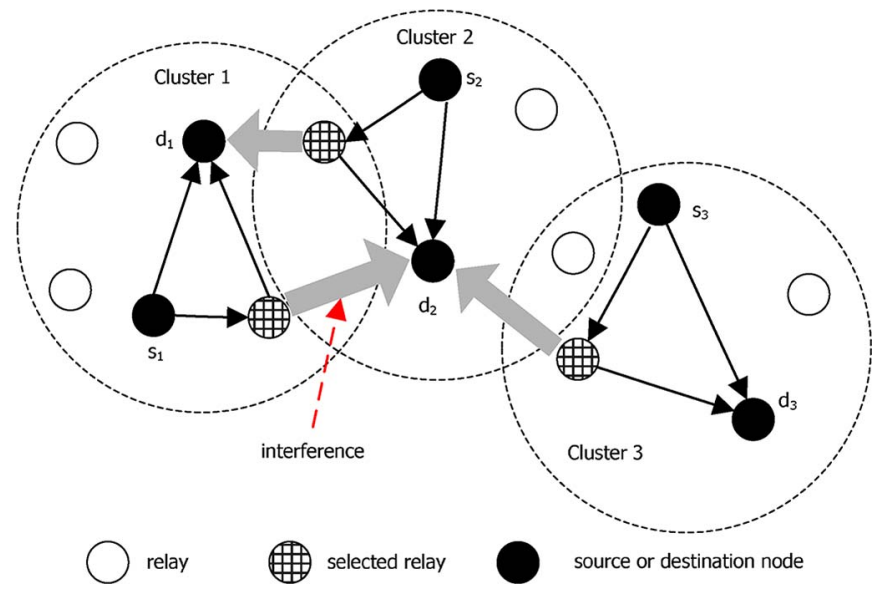

Fig. 1. Typical scenario of two-hop relay-assisted cooperative transmission. A cluster includes an S-D pair and the selected relay from candidates. The selected relay causes interference to other destinations.

$k_{i}$ are separated on two different channels, the simultaneous transmissions on channel 1 from source nodes in neighboring clusters will cause interference to $k_{i}$, along with those from the relays in the neighboring clusters on channel 2 to $d_{i}$.

We consider decode-and-forward (DF) relaying. The channels are assumed static during the whole relay selection process and are precisely estimated, and the channel estimation methods are discussed in Section VI-C. We also assume that there is no power control, i.e., the transmission power of each node is fixed. We first observe the interference-free SNR as the indication of the channel quality. In an isolated transmission cluster, the transmission of both source-to-relay and relay-todestination links has no interference. For specific S-D pair $\left(s_{i}, d_{i}\right)$ assisted by relay $k_{i}$, the received SNR of the sourceto-relay link is given by

$$
\gamma_{s_{i}, k_{i}}=\frac{P_{s_{i}}\left|h_{s_{i}, k_{i}}\right|^{2}}{N_{0}}
$$

where $P_{s_{i}}$ is the transmit power, $h_{s_{i}, k_{i}}$ is the channel gain between $s_{i}$ and $k_{i}$, and $N_{0}$ is the noise power. Similarly, the received SNR of the relay-to-destination link is given by

$$
\gamma_{k_{i}, d_{i}}=\frac{P_{k_{i}}\left|h_{k_{i}, d_{i}}\right|^{2}}{N_{0}} .
$$

We then analyze the signal-to-interference-plus-noise ratio (SINR) of each hop and the two-hop transmission rate.

\section{A. Source-to-Relay Link}

When interference from neighboring clusters is considered, the SINR of the source-to-relay link is

$$
\tilde{\gamma}_{s_{i}, k_{i}}=\frac{P_{s_{i}}\left|h_{s_{i}, k_{i}}\right|^{2}}{\sum_{j: j \neq i} P_{s_{j}}\left|h_{s_{j}, k_{i}}\right|^{2}+N_{0}} .
$$

Substituting (1) into (3), we get

$$
\tilde{\gamma}_{s_{i}, k_{i}}=\frac{\gamma_{s_{i}, k_{i}}}{\sum_{j: j \neq i} \gamma_{s_{j}, k_{i}}+1}
$$


and then, the rate of the source-to-relay link is

$$
c_{i}^{\mathrm{SR}}=\log \left(1+\tilde{\gamma}_{s_{i}, k_{i}}\right) .
$$

\section{B. Relay-to-Destination Link}

When interference from neighboring clusters is considered, the SINR of the relay-to-destination link is

$$
\tilde{\gamma}_{k_{i}, d_{i}}=\frac{P_{k_{i}}\left|h_{k_{i}, d_{i}}\right|^{2}}{\sum_{j: j \neq i} P_{k_{j}}\left|h_{k_{j}, d_{i}}\right|^{2}+N_{0}} .
$$

Although (3) and (6) have similar forms, (6) is more complicated since it depends on the selection decision $k_{j}$ of other S-D pairs. Substituting (2) into (6), respectively, we get

$$
\tilde{\gamma}_{k_{i}, d_{i}}=\frac{\gamma_{k_{i}, d_{i}}}{\sum_{j: j \neq i} \gamma_{k_{j}, d_{i}}+1} .
$$

The rate of the relay-to-destination link is

$$
c_{i}^{\mathrm{RD}}=\log \left(1+\tilde{\gamma}_{k_{i}, d_{i}}\right) .
$$

\section{Two-Hop Rate}

Because we consider DF relaying, the equivalent SINR for the S-D pair $\left(s_{i}, d_{i}\right)$, which is assisted by relay $k_{i}$, is given by

$$
\tilde{\gamma}_{d_{i}}=\min \left\{\tilde{\gamma}_{s_{i}, k_{i}}, \tilde{\gamma}_{k_{i}, d_{i}}\right\} .
$$

Assuming a unit system bandwidth on each channel, the achievable rate $c_{i}$ of this transmission cluster is

$$
\begin{aligned}
c_{i} & =\log \left(1+\tilde{\gamma}_{d_{i}}\right) \\
& =\log \left(1+\min \left\{\tilde{\gamma}_{s_{i}, k_{i}}, \tilde{\gamma}_{k_{i}, d_{i}}\right\}\right) \\
& =\min \left\{\log \left(1+\tilde{\gamma}_{s_{i}, k_{i}}\right), \log \left(1+\tilde{\gamma}_{k_{i}, d_{i}}\right)\right\}
\end{aligned}
$$

where we assume full-duplex relay; therefore, there is no $(1 / 2)$ coefficient in the rate expression. Note that making this assumption is for neat rate expressions, and as we restrict all transmissions being two-hop, the assumption does not change the nature of the relay selection problem.

\section{Problem Formulation}

We use matrix $\mathbf{A}_{N \times M}$ to represent the relay selection result. Each entry $a_{i r}$ denotes whether relay $r$ is selected by S-D pair $\left(s_{i}, d_{i}\right)$, i.e., we put integer constraints on $a_{i r}$ as follows:

$$
a_{i r}= \begin{cases}1, & \text { if } r \text { is assigned to }\left(s_{i}, d_{i}\right) \\ 0, & \text { otherwise. }\end{cases}
$$

Note that the selected relay for S-D pair $\left(s_{i}, d_{i}\right)$ satisfies $k_{i}=$ $\left\{r \mid a_{i r}=1\right\}$. The optimization problem to maximize the sum rate is formulated as

$$
\begin{array}{ll}
\text { maximize } & \sum_{i=1}^{N} c_{i} \\
\text { subject to } & \sum_{j=1}^{M} a_{i j}=1, \forall i \\
& \sum_{i=1}^{N} a_{i j} \leq 1, \forall j .
\end{array}
$$

The problem is complicated not only due to its combinational integer programming nature but also due to the SINR values.

If we look at the source-to-relay link SINR $\tilde{\gamma}_{s_{i}, k_{i}}$, the interference at relay $k_{i}$ is from the source nodes of neighboring clusters. Since these source nodes are predetermined, one can always find the optimal relay selection to maximize the sourceto-relay link rate, regardless of the relay selection of other S-D pairs.

However, the relay-to-destination link SINR $\tilde{\gamma}_{k_{i}, d_{i}}$ is much more complicated. The interference signal is from the relays of other clusters so that, when making the relay selection decision, the accurate impact of interference and the amount of interference itself that will be introduced to the network are unknown. Choosing the relay that maximizes the interferencefree SNR $\gamma_{k_{i}, d_{i}}$ can be a simple solution. However, the neighboring clusters may suffer severe interference by the choice of $k_{i}$, making the selection unfavorable from the network point of view. In the following analysis, we mainly focus on the optimization of the relay-to-destination link, addressing the potential interference induced by relays.

We see that, in this paper, we consider the interferencelimited scenario. For noise-limited scenario, relay selection has negligible impact on the rate of other S-D pairs; therefore, the relay selection is nothing but the isolated network problem [1], [7]-[9], [11]-[14]. For the interference-limited scenario, the interference power dominates and is much larger than the noise power, i.e., $\sum_{j: j \neq i} \gamma_{k_{j}, d_{i}} \gg 1$. Therefore, we can ignore the noise power as

$$
\tilde{\gamma}_{k_{i}, d_{i}} \approx \frac{\gamma_{k_{i}, d_{i}}}{\sum_{j: j \neq i} \gamma_{k_{j}, d_{i}}} .
$$

For the tractability of the analysis in this paper, we make a further approximation of the rate as

$$
c_{i}^{\mathrm{RD}}=\log \left(1+\tilde{\gamma}_{k_{i}, d_{i}}\right) \approx \log \left(\tilde{\gamma}_{k_{i}, d_{i}}\right)
$$

where the rational of this approximation is that the optimized selection of relays can lead to proper SINR so that ignoring 1 in the $\log$ operation will not lead to notable error. Moreover, this assumption is a conservative approximation of the link rate.

Substituting (13) into (14) leads to

$$
c_{i}^{\mathrm{RD}} \approx \log \gamma_{k_{i}, d_{i}}-\log \sum_{j: j \neq i} \gamma_{k_{j}, d_{i}} .
$$

The rates $c_{i}^{\mathrm{RD}}$ of clusters are coupled through the decision variable $a_{i j}$. An exhaustive search involves the following procedures. For a given selection of the relays for all S-D pairs, compute the rate of each cooperative link. Then, find the selection that maximizes the sum rate. Hence, the complexity is on the order of $M^{N}$, which is impossible for practical implementations. Moreover, S-D pairs are distributed and not aware of the channel conditions of other pairs. Hence, even for exhaustive search, there must be a central controller who collects the global CSI and informs each cluster the final decision. It extremely increases the signaling and processing cost. Therefore, we try to explore tractable solutions. 
A common solution is to set up a selection metric. Every S-D pair calculates the value of the metric and selects the relay with the maximum value. There have been some relay selection metrics such as "max-min"[15] and "harmonic mean" [8]. However, they are all derived for interference-free scenarios. To design a metric for networks with multiple concurrent transmissions, the impact of interference should first be decoupled. In the following, we decouple the impact of interference induced by multiple relays based on the expression in (15).

\section{DeCOUPling THE IMPACT OF INTERFERENCE ON THE RELAY-TO-DESTINATION LINKS}

As aforementioned, it is difficult to quantify the impact of interference induced by relay selection metrics such as $k_{j}$ on the rate of the link from relay $k_{i}$ to its associated destination $d_{i}$. That is because the second item in (15) always exerts a joint impact on the SINR. Here, we decouple this joint impact into a linear combination directly from the mathematical expression of the rate $c_{i}^{\mathrm{RD}}$. Specifically, we provide a lower bound and an upper bound of the relay-to-destination link rate, and they are both linear combinations of $\log \gamma_{k_{i}, d_{i}}$ (the $\log$ functions of the relay-to-destination link SNR), and the coefficients are also decoupled. Based on the analysis here, a metric is proposed in Section $\mathrm{V}$ by utilizing the commutative property of the addition operation, where the impact of the relay selection of each S-D pair is decoupled into its useful signal and the interference induced to other clusters.

We first show that the relay-to-destination link rate can be upper and lower bounded by a linear combination of $\log \gamma_{k_{j}, d_{i}}$. Then, we prove that the exact value of the rate can be further written with a linear combination of the two bounds. Assume that the number of neighboring clusters that cause interference to the S-D pair of interest $\left(s_{i}, d_{i}\right)$ is $L_{i}$.

Theorem 1-Lower Bound: For each S-D pair $\left(s_{i}, d_{i}\right)$, the rate $c_{i}^{\mathrm{RD}}$ defined in (15) can be lower bounded by a linear combination of the log functions of the SNRs as

$$
c_{i}^{\mathrm{RD}} \geq \log \gamma_{k_{i}, d_{i}}+\sum_{j: j \neq i} \alpha_{k_{j}, d_{i}} \log \gamma_{k_{j}, d_{i}}-\log L_{i}
$$

where

$$
\alpha_{k_{j}, d_{i}}=-\frac{\gamma_{k_{j}, d_{i}}}{\sum_{j^{\prime}: j^{\prime} \neq i} \gamma_{k_{j^{\prime}}, d_{i}}} .
$$

Proof: According to log-sum inequality, we have

$$
\sum_{j: j \neq i} \gamma_{k_{j}, d_{i}} \log \gamma_{k_{j}, d_{i}} \geq\left(\sum_{j: j \neq i} \gamma_{k_{j}, d_{i}}\right) \log \frac{\sum_{j: j \neq i} \gamma_{k_{j}, d_{i}}}{L_{i}}
$$

and thus

$$
\log \sum_{j: j \neq i} \gamma_{k_{j}, d_{i}} \leq \sum_{j: j \neq i} \frac{\gamma_{k_{j}, d_{i}}}{\sum_{j^{\prime}: j^{\prime} \neq i} \gamma_{k_{j^{\prime}}, d_{i}}} \log \gamma_{k_{j}, d_{i}}+\log L_{i} .
$$

The coefficients are still coupled with each other by the denominator. However, if $\sum_{j^{\prime}: j^{\prime} \neq i} \gamma_{k_{j^{\prime}}, d_{i}}$ is bounded for each S-D pair $\left(s_{i}, d_{i}\right)$, a relatively looser lower bound can be obtained. Then, the coefficient becomes

$$
\alpha_{k_{j}, d_{i}}=-\frac{\gamma_{k_{j}, d_{i}}}{B}
$$

where $B$ is the lower bound of $\sum_{j^{\prime}: j^{\prime} \neq i} \gamma_{k_{j^{\prime}}, d_{i}}$ for each $i$. For example, one can choose the sum of $L_{0}$ smallest $\gamma_{k_{j}}, d_{i}$, where $L_{0}=\min _{i} L_{i}$. This way, the coefficients are decoupled.

Theorem 2-Upper Bound: For each S-D pair $\left(s_{i}, d_{i}\right)$, the rate $c_{i}^{\mathrm{RD}}$ defined in (15) can be upper bounded by a linear combination of the log functions of SNRs as

$$
c_{i}^{\mathrm{RD}} \leq \log \gamma_{k_{i}, d_{i}}+\sum_{j: j \neq i} \beta_{k_{j}, d_{i}} \log \gamma_{k_{j}, d_{i}}-\log L_{i}
$$

where

$$
\beta_{k_{j}, d_{i}}=-\frac{1}{L_{i}}
$$

Proof: According to Jensen's inequality, we have

$$
\log \frac{\sum_{j: j \neq i} \gamma_{k_{j}, d_{i}}}{L_{i}} \geq \frac{\sum_{j: j \neq i} \log \gamma_{k_{j}, d_{i}}}{L_{i}}
$$

therefore

$$
\log \sum_{j: j \neq i} \gamma_{k_{j}, d_{i}} \geq \sum_{j: j \neq i} \frac{1}{L_{i}} \log \gamma_{k_{j}, d_{i}}+\log L_{i} .
$$

Substituting (24) into (15), we obtain the result.

The given two theorems indicate that the coefficients of the $\log$ functions are important for describing the impact of multiple interference sources. We now focus on a particular S-D pair $\left(s_{i}, d_{i}\right)$. For simplicity, we use $\alpha_{j}, \beta_{j}, \gamma_{j},(j \neq i)$ instead of $\alpha_{k_{j}, d_{i}}, \beta_{k_{j}, d_{i}}, \gamma_{k_{j}, d_{i}}$ and stack them into $1 \times L_{i}$ vectors $\boldsymbol{\alpha}, \boldsymbol{\beta}$, and $\boldsymbol{\gamma}$, respectively. Remark that $\boldsymbol{\alpha}$ and $\boldsymbol{\beta}$ provide the upper and lower bounds of the mathematical expression of the relay-to-destination link rate. They only require partial CSI. In the following, we show that, by combining the coefficients of the upper and lower bounds, a new coefficient vector can be obtained, which renders the accurate rate in (15).

Corollary 1: For specific transmission cluster $i$, there exists a $1 \times L_{i}$ vector $\pi$ such that the rate defined in (15) can be written in a decoupled expression of interference, i.e., specifically, a linear combination of the log functions of the SNRs as

$$
c_{i}^{\mathrm{RD}}=\log \gamma_{i}+\sum_{j: j \neq i} \pi_{j} \log \gamma_{j}-\log L_{i}
$$

such that $\boldsymbol{\pi}=\mu \boldsymbol{\alpha}+\nu \boldsymbol{\beta}$, where $\mu+\nu=1$ and $\boldsymbol{\pi} \cdot \boldsymbol{e}=-1$, with $e$ being an $L_{i} \times 1$ vector with all elements equal to 1 .

Proof: We first define

$$
\tilde{c}_{i}=c_{i}^{\mathrm{RD}}+\log L_{i}-\log \gamma_{i}
$$

From the rate bounds obtained in (16) and (21), we have

$$
\begin{aligned}
& \boldsymbol{\alpha} \cdot \log \gamma \leq \tilde{c}_{i} \\
& \boldsymbol{\beta} \cdot \log \gamma \geq \tilde{c}_{i} .
\end{aligned}
$$


Therefore

$$
\begin{aligned}
& \boldsymbol{\alpha} \cdot \log \boldsymbol{\gamma}=\varepsilon_{1} \tilde{c}_{i} \\
& \boldsymbol{\beta} \cdot \log \boldsymbol{\gamma}=\varepsilon_{2} \tilde{c}_{i}
\end{aligned}
$$

where $\varepsilon_{1} \leq 1$, and $\varepsilon_{2} \geq 1$.

Through some simple calculations, let $\mu=\left(1-\varepsilon_{2}\right) /\left(\varepsilon_{1}-\right.$ $\left.\varepsilon_{2}\right)$, and $\nu=\left(1-\varepsilon_{1}\right) /\left(\varepsilon_{2}-\varepsilon_{1}\right)$. Then, the conditions are satisfied.

Then, as aforementioned, we use $\alpha_{j}, \beta_{j}$, and $\gamma_{j}(j \neq i)$ instead of $\alpha_{k_{j}, d_{i}}, \beta_{k_{j}, d_{i}}$, and $\gamma_{k_{j}, d_{i}}$, and stack them into vectors $\boldsymbol{\alpha}, \boldsymbol{\beta}$, and $\boldsymbol{\gamma}$, respectively. We, therefore, have

$$
\begin{aligned}
\boldsymbol{\pi} \cdot \boldsymbol{e} & =\mu \sum_{j: j \neq i} \alpha_{j}+\nu \sum_{j: j \neq i} \beta_{j} \\
& =-\mu \sum_{j: j \neq i} \frac{\gamma_{k_{j}, d_{i}}}{\sum_{j^{\prime}: j^{\prime} \neq i} \gamma_{k_{j^{\prime}}, d_{i}}}-\nu \sum_{j: j \neq i} \frac{1}{L_{i}} \\
& =-\mu-\nu=-1
\end{aligned}
$$

where $L_{i}$ is the number of transmission clusters that cause interference to S-D pair $\left(s_{i}, d_{i}\right)$; thus, $L_{i}=\sum_{j: j \neq i} 1$.

Note that the coefficients $\mu$ and $\nu$ are not identical for different clusters, and they also depend on the relay selection result of other clusters. To have a purely decoupled relay selection metric, we will propose a heuristic identical coefficient setting for the linear combination of lower and upper bounds (16) and (21) in the following.

\section{Relay SELECTION METRic For DECODE-AND-FORWARD WITH INTERFERENCE}

\section{A. Baseline Relay Selection Scheme}

The conventional "max-min" relay selection metric is used in interference-free configurations, i.e., from the S-D pair $i$ 's point of view, we have

$$
k_{i}^{*}=\underset{k_{i} \in \mathcal{R}}{\arg \max } \min \left\{\gamma_{s, k}, \gamma_{k, d}\right\}
$$

and will be compared with our proposed interference-aware relay selection schemes in the simulations. The "max-min" criterion is an efficient selection metric for both $\mathrm{AF}$ and $\mathrm{DF}$ relaying schemes. In particular, it is the optimal solution for DF protocols. In addition, it simplifies the implementation complexity.

\section{B. Relay Selection for the Source-to-Relay Link}

Taking the interference from other sources into consideration, from the S-D pair $i$ 's point of view, the metric for the source-to-relay link should be modified as follows:

$$
k_{i}^{*}=\underset{k_{i} \in \mathcal{R}}{\arg \max }\left\{\tilde{\gamma}_{s_{i}, k_{i}}\right\}=\underset{k_{i} \in \mathcal{R}}{\arg \max }\left\{\frac{\gamma_{s_{i}, k_{i}}}{\sum_{j: j \neq i} \gamma_{s_{j}, k_{i}}+1}\right\} .
$$

In [15], a similar metric is designed on the consideration of outage probability.

\section{Relay Selection for the Relay-to-Destination Link}

Based on the SINR of the relay-to-destination link obtained in interference-limited networks, the relay selection metric could be

$$
k_{i}^{*}=\underset{k_{i} \in \mathcal{R}}{\arg \max }\left\{\tilde{\gamma}_{k_{i}, d_{i}}\right\} .
$$

However, $\tilde{\gamma}_{k_{i}, d_{i}}$ depends on the selection decisions of other links. Fortunately, based on the decoupling feature of the interference, particularly the resultant Corollary 1 in Section IV, the rate $c_{i}^{\mathrm{RD}}$ can be written as a linear combination of the $\log$ functions of the SNRs. The sum-rate objective in (12) can then be written as a linear combination of the log functions of the SNRs. According to the commutative property of the summation operation, we can rearrange the items of the linear combination and decouple the contribution of the relay selection of each S-D pair into its useful signal strength and the possible interference induced to other pairs.

First, we define global parameter $p$ (independent of $i$ ) for combining the lower and upper bounds, and the resultant coefficients of $\log \gamma_{k_{i}, d_{j}}$ are

$$
\xi_{k_{i}, d_{j}}=p \alpha_{k_{i}, d_{j}}+(1-p) \beta_{k_{i}, d_{j}} .
$$

Then, the existence of $p^{*}$ that precisely describe the system sum rate is shown as follows.

Lemma $1-$ Existence of $p^{*}$ : If the relay selection result $\left\{k_{1}^{*}, k_{2}^{*}, \ldots, k_{N}^{*}\right\}$ maximizes the sum rate, i.e.,

$$
\left\{k_{1}^{*}, k_{2}^{*}, \ldots, k_{N}^{*}\right\}=\underset{k_{i} \in \mathcal{R}}{\arg \max } \sum_{i} c_{i}^{\mathrm{RD}}
$$

there exists a unique constant $p^{*} \in(0,1)$ such that

$\sum_{i}\left(\log \gamma_{k_{i}^{*}, d_{i}}+\sum_{j: j \neq i} \xi_{k_{j}^{*}, d_{i}} \log \gamma_{k_{j}^{*}, d_{i}}-L_{i}\right)=\max _{k_{i} \in \mathcal{R}} \sum_{i} c_{i}^{\mathrm{RD}}$

where, $\forall i, \xi_{k_{i}^{*}, d_{i}}=p^{*} \alpha_{k_{j}, d_{i}}+\left(1-p^{*}\right) \beta_{k_{j}, d_{i}}$.

Proof: The left-hand side of (39) is a function of $p$, which is denoted by $f(p)$. For a fixed selection $\left\{k_{1}^{*}, k_{2}^{*}, \ldots, k_{N}^{*}\right\}$, the rate has a constant value. $f(p)$ is a monotonic continuous function of $p$, with $f(p=0) \geq \max \sum_{i} c_{i}^{\mathrm{RD}}$ and $f(p=1) \leq$ $\max \sum_{i} c_{i}^{\mathrm{RD}}$, due to the lower and upper bound properties from Theorems 1 and 2 . Therefore, there must be an unique constant $p^{*} \in(0,1)$ that satisfies (39).

This lemma can be regarded as a network-wise version of Corollary 1 , where Corollary 1 is for a specific transmission cluster.

With the commutative property of summation operation, we can rewrite the left-hand side of (39) as

$$
\sum_{i}\left(\log \gamma_{k_{i}^{*}, d_{i}}+\sum_{j: j \neq i} \xi_{k_{i}^{*}, d_{j}} \log \gamma_{k_{i}^{*}, d_{j}}-L_{i}\right)
$$

and notice that the items inside the brackets only depend on the relay selection decision $k_{i}^{*}$ of S-D pair $\left(s_{i}, d_{i}\right)$. Therefore, by selecting appropriate $p$, with the resulting coefficients $\xi_{k_{i}, d_{j}}$ 
given by (37), the relay selection metric is decoupled into each S-D pair $i$, i.e., S-D pair $i$ seeks to select the relay as

$$
k_{i}^{*}=\underset{k_{i} \in \mathcal{R}}{\arg \max }\left\{\log \gamma_{k_{i}, d_{i}}+\sum_{j: j \neq i} \xi_{k_{i}, d_{j}} \log \gamma_{k_{i}, d_{j}}\right\}
$$

where $L_{i}$ is eliminated since it does not depend on the relay selection. We can see that $p$ plays an important role for the network performance. Some discussions regarding the selection of $p$ are listed as sequel.

- If $p=0$, the selection metric aims to maximize the upper bound of the sum rate.

- If $p=1$, the selection metric aims to maximize the lower bound of the sum rate.

- Generally, parameter $p$ should be selected according to the network condition, and its impact on the system performance will be discussed in detail with simulation results. Although Lemma 1 states that, given the relay selection result, there exists a unique $p$, with which we can precisely express the sum rate in a decoupled way. However, generally, the inverse does not hold. In other words, if there is a genie-aided optimal relay selection result $\left\{k_{1}^{*}, k_{2}^{*}, \ldots, k_{N}^{*}\right\}$, one can get the corresponding $p^{*}$. However, using the relay selection metric (41), given by this $p^{*}$, does not guarantee the same relay selection result. This is because using $p^{*}$ only precisely describes the sum rate with the relay selection combination $\left\{k_{1}^{*}, k_{2}^{*}, \ldots, k_{N}^{*}\right\}$, whereas it cannot predict the sum rate when other candidate relays are selected. Nevertheless, the lower and upper bounds always hold; thus, using a unique $p$ to generate all $\xi_{k_{i}, d_{j}}$ is a reasonable heuristic. Its performance will be evaluated in the simulations.

- We will see that the optimal $p$ is generally small, i.e., the upper bound is favorable. Comparing $\alpha_{k_{j}, d_{i}}$ and $\beta_{k_{j}, d_{i}}$ in (22) and (20), we can see that $\beta_{k_{j}, d_{i}}$ is in fact a rough approximation of $\alpha_{k_{j}, d_{i}}$, where we have

$$
\mathbb{E}\left\{\frac{\gamma_{k_{j}, d_{i}}}{\sum_{j^{\prime}: j^{\prime} \neq i} \gamma_{k_{j^{\prime}}, d_{i}}}\right\} \geq \frac{\mathbb{E}\left\{\gamma_{k_{j}, d_{i}}\right\}}{\mathbb{E}\left\{\sum_{j^{\prime}: j^{\prime} \neq i} \gamma_{k_{j^{\prime}}, d_{i}}\right\}}=\frac{1}{L_{i}}
$$

where $\mathbb{E}\{\cdot\}$ is the expectation operator, and the last equality holds when the transmission power of the relays is the same. Although $\beta_{k_{j}, d_{i}}$ is dominate for determining $\xi_{k_{i}, d_{j}}$, a slight change of $p$ can still have notable impact on system performance. In conclusion, setting $p$ is nontrivial.

\section{Proposed Relay Selection Metric}

Combining the source-to-relay link metric (35) and relayto-destination link metric (41), our relay selection metric for networks with interference finally becomes

$$
\min \left\{\log \gamma_{s_{i}, k_{i}}, \log \gamma_{k_{i}, d_{i}}\right\}+\sum_{j: j \neq i} \xi_{k_{i}, d_{j}} \log \gamma_{k_{i}, d_{j}} .
$$

It is interesting to view the physical meaning of the metric: The first term corresponds to the equivalent two-hop transmission rate and is identical to the conventional "min-max" metric (34). The second item in (43) represents the penalty ${ }^{1}$ paid for the interference induced by a selected relay to other clusters, which is the unique feature of our interference-aware relay selection metric.

The metric represents the relay selection preferences of S-D pairs themselves. However, when two pairs' preferences conflict, the selection should be globally optimized. Now, the objective of relay selection is to optimize the following summation of the metric as

$$
\begin{aligned}
\max _{\left\{k_{1}, k_{2}, \ldots, k_{N}\right\}} \sum_{i}\left(\min \left\{\log \gamma_{s_{i}, k_{i}}, \log \gamma_{k_{i}, d_{i}}\right\}\right. \\
\left.+\sum_{j: j \neq i} \xi_{k_{i}, d_{j}} \log \gamma_{k_{i}, d_{j}}\right) .
\end{aligned}
$$

The given problem is in fact a bipartite graph matching problem, where the metric value acts similar to the weight on the edge connecting an S-D pair and a candidate relay. Algorithms for relay selection, i.e., both centralized and distributed, are proposed in the following.

\section{Relay SElection Algorithms}

With the proposed relay selection metric, when two or more S-D pairs' best candidate relay conflicts, the duty of the relay selection algorithm is to choose the relay for each pair that is best for the sum rate (12). We formulate the relay selection as a weighted bipartite matching problem [22]. Recall that we denote all S-D pairs as set $\mathcal{P}$ (with $N$ elements) and all candidate relays as set $\mathcal{R}$ (with $M$ elements). Each element in $\mathcal{P}$ and $\mathcal{R}$ is a vertex in the graph. There is an edge between any two elements, one of which is from $\mathcal{P}$, and the other is from $\mathcal{R}$. Corresponding to (43), the weight of each edge between $i \in \mathcal{P}$ and $r \in \mathcal{R}$ is

$$
w_{i, r}=\min \left\{\log \gamma_{s_{i}, r}, \log \gamma_{r, d_{i}}\right\}+\sum_{j: j \neq i} \xi_{r, d_{j}} \log \gamma_{r, d_{j}} .
$$

where we use $r$ for candidate relays to distinguish from the selected relay $k_{i}$ used earlier. Finally, the bipartite graph $G$ is denoted as $G=(\mathcal{P} \cup \mathcal{R}, \mathcal{P} \times \mathcal{R})$ [22], where $\mathcal{P} \cup \mathcal{R}$ represents all vertices, and $\mathcal{P} \times \mathcal{R}$ represents all edges. We also denote matrix $\mathbf{W}_{N \times M}$ as the weight matrix, of which the elements are $w_{i, r}$. For example, assume that there are four candidate relays for three S-D pairs to choose, and the weight matrix is assumed as follows:

$$
\mathbf{W}=\left(\begin{array}{llll}
4 & 4 & 3 & 0 \\
1 & 2 & 4 & 1 \\
0 & 3 & 4 & 3
\end{array}\right)
$$

Fig. 2 shows the corresponding bipartite graph of this particular system. There is a weighted line between each S-D pair and relay. At most, one link is allowed to be attached on each node in the final matching decision.

\footnotetext{
${ }^{1}$ It is a penalty since $\xi_{k_{i}, d_{j}}$ is always negative
} 


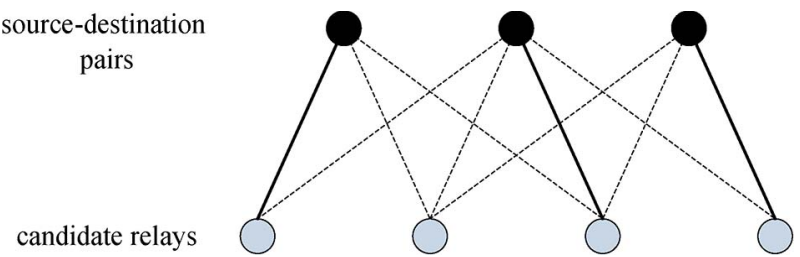

Fig. 2. Relay selection bipartite graph, where the solid lines represent the relay selection decisions.

To incorporate the distributed design of relay selection, we define the following relevant states as the terminology in matching games [20]:

- ground state (GS: state with the maximal utility);

- optimal stable state (OSS: stable state with the maximal utility).

The first state is global optimal, but there is no reason why selfish S-D pairs should be able to find it distributively and do not move away from it once reached. Only a centralized controller can help S-D pairs in such a profitable way. The second state (OSS) is a constrained optimal state; it will not change once reached. Here, we present methods to reach these two states with centralized and distributed algorithms, respectively.

\section{A. Centralized Scheme Based on GS}

The Hungarian algorithm [22], which is a common approach to weighted bipartite problems, is used to solve the centralized relay selection. The standard Hungarian algorithm requires $M=N$. However, if $N \leq M$, we can easily introduce $M-N$ virtual S-D pairs, from which the weights to each relay are set small enough, i.e., smaller than the minimum of the weights from all the real S-D pairs to the relays. The computational complexity of the Hungarian algorithm is $O\left(M^{3}\right)$, and it requires significant signaling overhead or, at least, a central controller. For the example shown in Fig. 2, a perfect matching is demonstrated by the solid lines in Fig. 2. The globally maximum weight sum is $w_{1,1}+w_{2,3}+w_{3,4}=11$.

\section{B. Distributed Scheme Based on Stable State}

With the proposed selection metric in (43), a distributed implementation is carried out here, which is based on the concept of stable state. We first provide the formal definition of stable and unstable relay selection and then prove the existence of the stable selection with the distributed relay selection algorithm.

Definition 1-Unstable Selection: A selection result $\mathbf{A}$ is unstable if $\exists r, r^{\prime} \in \mathcal{R}$ and $\exists i, i^{\prime} \in \mathcal{P}$; $i$ selects $r$ and $i^{\prime}$ selects $r^{\prime}$, although $w_{i, r^{\prime}}>w_{i, r}$ and $w_{i^{\prime}, r}>w_{i^{\prime}, r^{\prime}}$.

Definition 2-Stable Selection: If a selection is not unstable, it is a stable selection.

Theorem 3-Existence of Stable Selection: There always exists a stable selection for our relay selection problem.

Proof: This is a direct result for the stable marriage problem [23]. A constructive proof is as follows, which is based on the deferred acceptance procedure (DAP) algorithm in the traditional marriage problem [23]. We employ it in our case and summarize it in Algorithm 1.

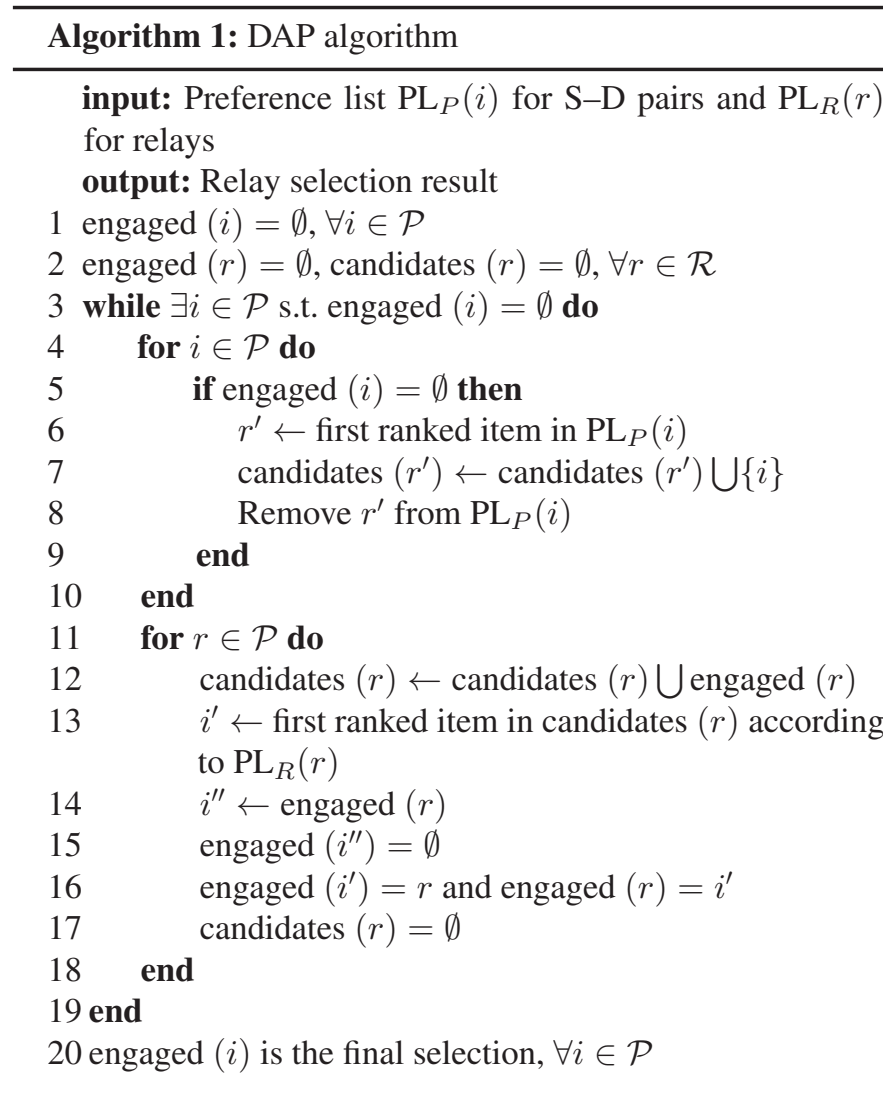

First, each S-D pair $i$ or relay $r$ keeps preference list $\mathrm{PL}_{P}(i)$ or $\mathrm{PL}_{R}(r)$, respectively. Taking an $\mathrm{S}-\mathrm{D}$ pair $i$ for example, all the relays are stored in $\mathrm{PL}_{P}(i)$ with the descending order of the associated weights, i.e., the first item in the list is the most favorite relay of the S-D pair. Then, each $\mathrm{S}-\mathrm{D}$ pair $i$ has a list engaged $(i)$ indicating its selected relay. Each relay $r$ has a list engaged $(r)$ indicating the $\mathrm{S}-\mathrm{D}$ pair it agrees to be selected. Each relay $r$ also keeps a list candidate $(r)$, which includes all the $\mathrm{S}-\mathrm{D}$ pairs that have proposed to select it.

The iteration in the algorithm terminates when all the S-D pairs are labeled as "engaged," i.e., a relay has been selected for each of them.

In the first group of iterations (lines 4-10), each S-D pair, without being engaged, proposes to its favorite relay from $\mathrm{PL}_{P}(i)$. The proposal is stored in the candidate list of the corresponding relay (line 7). Accepted S-D pairs are "engaged" and will not propose. To avoid proposing the same relay in the next iteration if the proposal is rejected, the proposed relay is removed from its preference list (line 8).

In the second group of iterations (lines 11-18), each relay selects its favorite S-D pair from the already "engaged" one and those that proposes (stored in the candidates list) this time. The winner will be the new "engaged" one. If the winner is different from the previously engaged S-D pair, the original S-D pair is reset to "not engaged" (lines 14-16).

As the algorithm terminates, no relay will deviate to a more favorable relay, and no S-D pair can select a better relay without switching another S-D pair to a worse selection; therefore, the system is stable. 
As proven in [23], the given algorithm has the complexity of $O(M N)$. It can be briefly explained as follows. First, $N$ $\mathrm{S}-\mathrm{D}$ pairs propose to select their most favorite relays. Then, any relay may turn to a more favorable S-D pair, which will happen at most $N-1$ times as if the relay tries all the S-D pairs. In addition, since there are $M$ relays, the worst case is $N+M(N-1)$ iterations; therefore, the complexity is on the order of $M N$.

Although DAP could not guarantee the OSS [20], it is still an efficient way to find a stable relay selection. We will compare the distributed relay selection algorithm with the centralized relay selection algorithm (based on the Hungarian algorithm) in the simulations.

\section{Overall Flow of Distributed Relay Selection}

The complete distributed relay selection procedure is summarized as follows.

1) The S-D pairs should calculate the weight (43) with each candidate relay. The proposed metric relies on the CSI from the relay to the neighboring destinations. Therefore, the channel estimation is performed first.

- In-cluster channel: $\gamma_{k_{i}, d_{i}}$ can be estimated at destination $d_{i}$, and relays receive this information through feedback.

- Cross-cluster channel: Channel information such as $\gamma_{k_{i}, d_{j}}$ can also be obtained through feedback between clusters. However, if we exploit the channel reciprocity, the relay can estimate the channel from the destinations and use its transpose. This kind of estimation does not need to be explicit, and relays can opportunistically listen to the channel when the destinations are transmitting if two-way communications exists.

- Far-away cluster: Since a relay will only elevate effective interference in a certain range, the interference caused by far-away clusters can be ignored.

2) When the metric weight (43) is ready at each S-D pair, e.g., stored at the destination, the destination will send the weight to the candidate relay. This requires $M N$ transmissions of a real number. Then, the candidate relay will store these weights in its preference list.

3) As Algorithm 1 starts, the proposal from a S-D pair for selecting a relay requires transmission of a 1-bit information, and upon receiving it, the relay will send back another 1-bit information indicating acceptance or reject. This requires $2 N$ transmissions of the 1-bit information. Finally, as lines 11-18 of Algorithm 1 show, the relay may readapt its "engaged" S-D pair. According to the complexity analysis, this will include at most $2 M(N-$ 1) 1-bit information transmissions.

To conclude, in addition to the channel estimation overhead, the signaling overhead of the distributed relay selection scheme includes $M N$ real number transmissions and approximately $2[N+M(N-1)]$ 1-bit information transmissions. In practice, this can be coordinated by synchronized round-robin polling before the data transmission starts.

\section{Existence of the Direct Link}

If the direct link is taken into account, the algorithm can be adapted under two different scenarios, where we take fullduplex relay for example.

- In one case, the S-D pair either uses a relay for two-hop transmission or uses the direct link alone. We can construct a virtual relay to represent the direct link for each S-D pair as one of its candidates. The virtual relay has no second-hop interference to other destinations. In this case, the weight of selecting direct link can simply be $\log \gamma_{s, d}$, where $\gamma_{s, d}$ is the direct link SINR.

- In the second case, the destination will combine the signal received from the direct link and from the relay if selected. Here, the direct link is always utilized. Using the maximal ratio combining scheme, the rate $c_{i}$ for S-D pair $i$ in (10) should be

$$
\log \left(1+\gamma_{s, d}+\min \left\{\tilde{\gamma}_{s_{i}, k_{i}}, \tilde{\gamma}_{k_{i}, d_{i}}\right\}\right) .
$$

However, here, the decoupling property does not hold even if we use the approximation in (43). However, as a conservative approximation, metric (43) can be adapted as

$$
\log \left(\gamma_{s, d}+\min \left\{\gamma_{s_{i}, k_{i}}, \gamma_{k_{i}, d_{i}}\right\}\right)+\sum_{j: j \neq i} \xi_{k_{i}, d_{j}} \log \gamma_{k_{i}, d_{j}} .
$$

The case for half-duplex relay with a direct link is more complicated and will be left for our future work.

\section{Simulations AND Discussions}

Here, the performance of the proposed relay selection metric and the corresponding algorithms are validated through computer simulations. The simulation settings follow the system model in Section II. The default simulation topology is generated as shown in Fig. 3(a): We first generate a square grid with $4 \times 4$ grid nodes. Within the grid, there are nine squares, of which the side length is denoted by $S_{l}$. We randomly place one S-D pair in each square, and the distance between each source and its corresponding destination is $100 \mathrm{~m}$. We then place one candidate relay randomly in a circular area with radius $0.5 S_{l}$ centering at each grid node. In Fig. 3(a), we provide two exemplary circular areas with a dotted line as their border. As a result, there are 16 candidate relays. We also simulate more random topologies, as depicted in Fig. 3(b), in which the S-D pairs are randomly distributed in the whole square area with each side $3 S_{l}$, and the relays are placed in the same way, except that the center of the circular is randomly placed in the whole square area. For both topologies, we can vary the side length of each square to investigate the impact of interference level. By decreasing the side length, the network becomes denser and, thus, more interference limited. We apply the lognormal shadowing channel model [21] to take both path loss and shadowing effect into account. Fading is not considered. Note that, although 


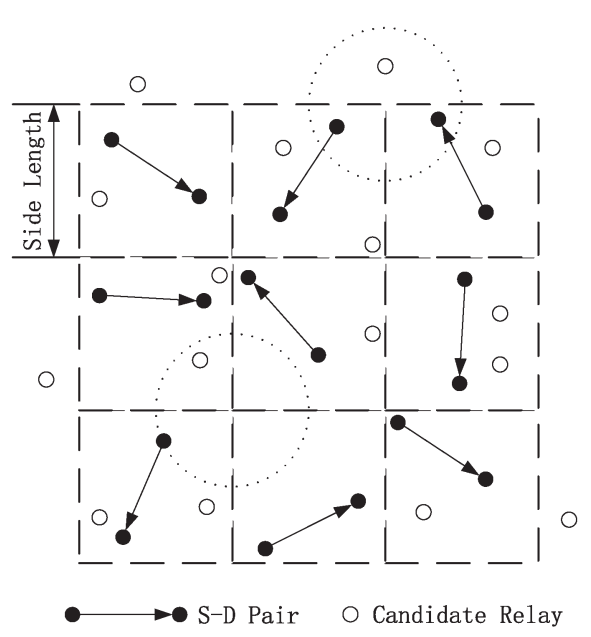

(a)

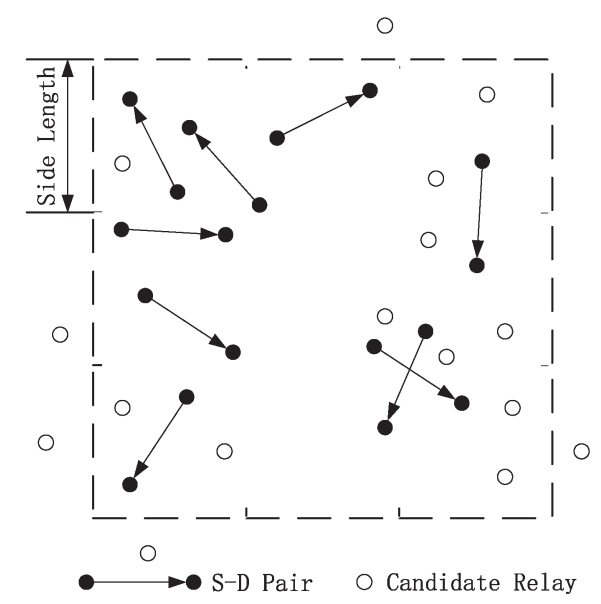

(b)

Fig. 3. Example of the simulation topologies. (a) Default simulation topology. (b) More random simulation topology.

TABLE I

SiMULATION PARAMETERS

\begin{tabular}{l|r}
\hline Parameter & Value \\
\hline reference distance $\left(d_{0}\right)$ & $1 \mathrm{~m}$ \\
channel gain at reference distance & $30 \mathrm{~dB}$ \\
path-loss exponent $(n)$ & 3 \\
shadowing standard deviation $(\sigma)$ & $7 \mathrm{~dB}$ \\
bandwidth $(B)$ & $5 \mathrm{MHz}$ \\
side length & $100 \mathrm{~m}$ \\
no. of interfering clusters to each S-D pair $\left(L_{i}\right)$ & 8 \\
\hline
\end{tabular}

approximation is made in (14) for the ease of analysis, in the simulations, all the SINRs are calculated according to their true value without any approximation. Unless otherwise specified, all the curves about our proposed interference-aware metric are based on the distributed relay selection algorithm, and the default simulation topology is used according to Fig. 3(a). We simulate 300 randomly generated topologies for each set of parameters. The default simulation parameters are summarized in Table I.

First, the performance improvement is verified in Fig. 4. Four curves, representing the conventional max-min metric, the interference-aware metric with fixed $p=10^{-4}$, the interference-aware metric with optimal $p$, and the optimal se-

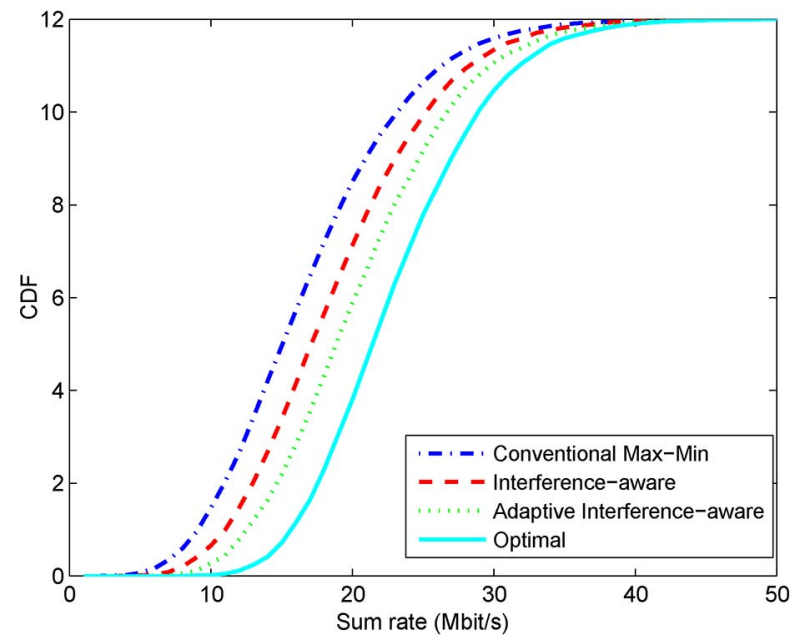

Fig. 4. Cumulative distribution function of the network sum rate with different relay selection metrics. For "interference-aware," we use fixed $p=10^{-4}$, whereas for "adaptive interference-aware," we search for the optimal $p$. The optimal is found by exhaustive search.

lection by exhaustive search, ${ }^{2}$ are demonstrated. The optimal result pushes forward the average sum rate by $35 \%$ over the conventional max-min metric. It shows that the conventional max-min metric is not efficient, particularly in interferencelimited scenarios. The interference-aware metric with adaptive $p$ improves the performance by $25 \%$. It is obtained by numerically optimizing the selection of $p$. Even with $p$ fixed at $p=10^{-4}$, our interference-aware metric still outperforms conventional max-min metric by $15 \%$.

In this set of simulations, we focus on the outage probability. In Fig. 4, we can see that the average rate per S-D pair is larger than $2 \mathrm{Mb} / \mathrm{s}$. However, as will be shown in Fig. 7, the rates of different clusters are unfair; therefore, there exist some links with very low rates. Outage occurs when an S-D pair cannot achieve the target transmission rate, whichever relay the pair selects. The threshold rate is $5 \mathrm{~kb} / \mathrm{s}$ in our settings. As shown in Fig. 5, about $7 \%-10 \%$ of the S-D pairs are in outage. In addition, note that, in the interference-limited scenarios, increasing transmit power dose not always improve outage probability. This is because, when transmit power is high enough, interference rather than noise dominates the SINR, and the interference level also increases with the useful signal strength.

Next, we show how the level of interference affects the sum rate. Fig. 6 is obtained by varying the side length of the grid squares. Both default topology and more random topology are simulated, and the parameter $p$ for our interference-aware metric is optimized, as shown in Table II. For both kinds of topologies, the sum rate increases as the side length $S_{l}$ becomes larger as expected. Moreover, the proposed metric outperforms the conventional metric by a constant rate gap because when $S_{l}$ grows large enough, we can treat each S-D pair as isolated. However, in the more random topology, some S-D pairs and corresponding relays could be very close to each other, even

\footnotetext{
${ }^{2}$ We ignore far-away relays from an S-D pair so that the complexity is reduced.
} 


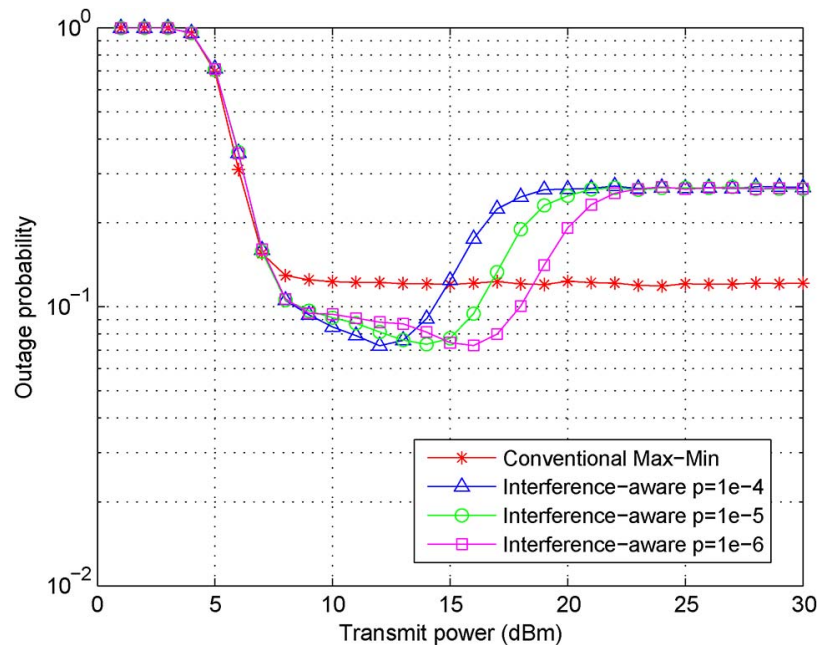

Fig. 5. Outage probability with different relay selection metrics. The threshold rate is $5 \mathrm{kbit} / \mathrm{s}$. Although as Fig. 4 shows, the average rate is high, and the rates of different clusters are unfair; therefore, there exist some links with very low rate.

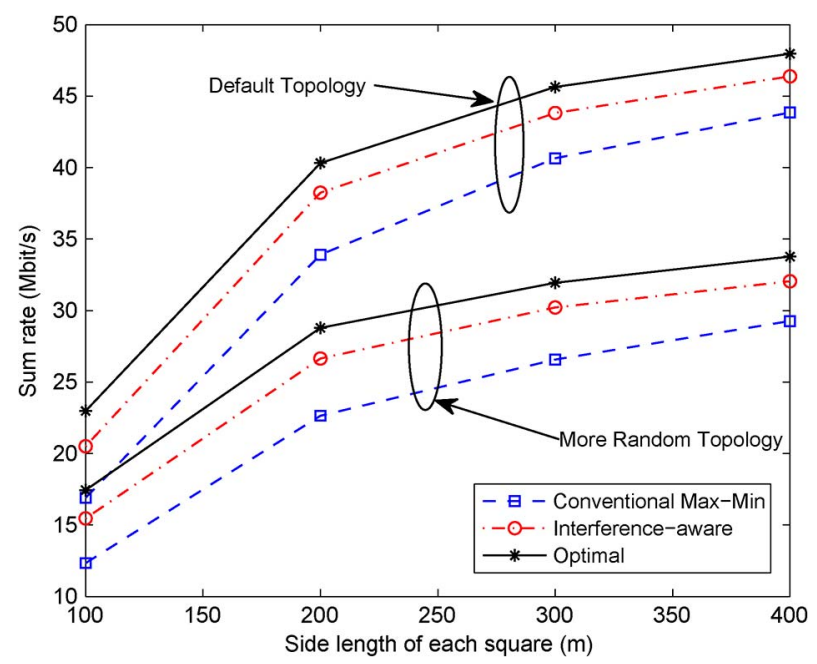

Fig. 6. Impact of interference on the sum rate. By decreasing the side length of each square, the network becomes denser and, thus, more interference limited. Two sets of topologies are simulated.

TABLE II

AVERage VALue of Optimal $p$

\begin{tabular}{c|c|c}
\hline Side Length $(\mathrm{m})$ & Default Topology & More Random Topology \\
\hline 100 & $18.87 \times 10^{-4}$ & $4.894 \times 10^{-4}$ \\
200 & $68.68 \times 10^{-4}$ & $10.43 \times 10^{-4}$ \\
300 & $109.3 \times 10^{-4}$ & $17.79 \times 10^{-4}$ \\
400 & $161.5 \times 10^{-4}$ & $24.43 \times 10^{-4}$ \\
\hline
\end{tabular}

when the average node density is low; therefore, the average rate is lower than the case in the default topology. In addition, note that, with a square side length of $100 \mathrm{~m}$, as we have nine $\mathrm{S}-\mathrm{D}$ pairs, the average per $\mathrm{S}-\mathrm{D}$ pair rate is around $2 \mathrm{Mb} / \mathrm{s}$ (about $1.5 \mathrm{Mb} / \mathrm{s}$ for more random topology); thus, the average spectral efficiency is around $0.4 \mathrm{~b} / \mathrm{s} / \mathrm{Hz}(0.3 \mathrm{~b} / \mathrm{s} / \mathrm{Hz}$ for more random topology). In this case, the approximation condition of high SINR in (14) actually does not hold while we still achieve substantial gain over the conventional metric.

Based on Fig. 4 and Table II, we summarize the impact of SNR or interference level on choosing appropriate values

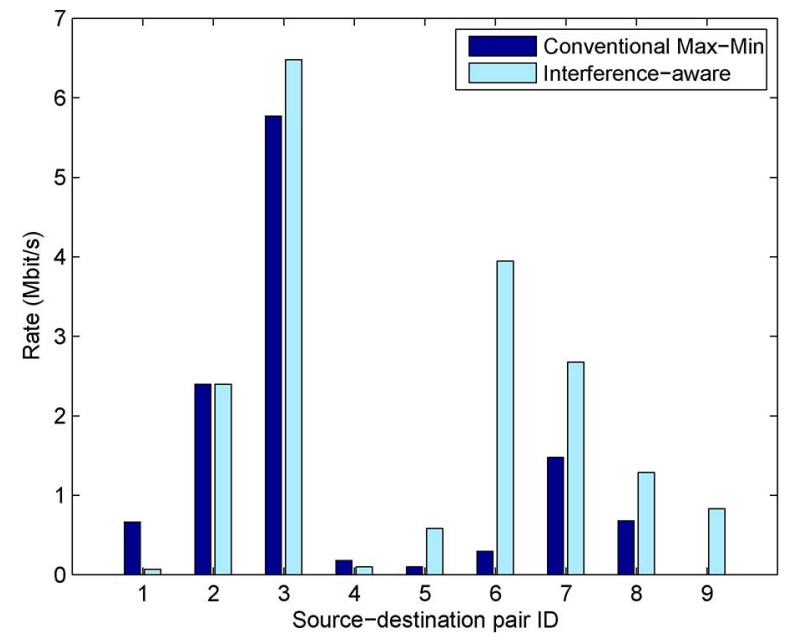

Fig. 7. Snapshot of transmission rate for a specific topology with nine S-D pairs, labeled by ID $1-9$.

of $p$. First, the interference-aware metric with fixed $p$ is not always better than the conventional metric. Second, the optimal $p$ depends on the average transmit power. As shown in Fig. 4, there exists an effective region for a specific value of $p$, and the curve is in a "U" shape. This is because the precision of the bounds depends on the SNR values. As the transmit power increases (interference level increases), the optimal $p$ shifts to other values; thus, the outage probability may increase for a given $p$. Specifically, for larger transmit power, we tend to choose a smaller $p$ to get better performance. The value of $p$ is often small because the lower bound is much looser than the upper bound. Third, we provide the optimal $p$ under different node densities (equals to $1 / S_{l}^{2} \mathrm{~m}^{-2}$, where $s_{l}$ is the side length) in Table II. Similar to what we have observed in Fig. 4, with larger node density, i.e., smaller side length, the interference level increases, and the optimal $p$ decreases. In addition, high randomness also requires smaller $p$, which is due to severe interference scenarios that happen more often. The table can serve as a guideline for the choice of optimal $p$ under different node densities, with moderate-to-high node distribution randomness. Conservatively, a small $p$ around $10^{-4}$ to $10^{-3}$ can be chosen in most cases because these values work well under a high interference level, whereas under low interference or low SNR conditions, the differences among the performances of various $p$ values are small, as shown in the low SNR regime in Fig. 4.

A snapshot of the cluster rates for one topology is demonstrated in Fig. 7. We remark that, although our interferenceaware metric tries to reduce the interference caused to others, it is not a fairness-guaranteed criterion since the optimization objective in (12) is to maximize the sum rate. Some relay selection decision may benefit the sum rate; however, it may cause very low rate for some S-D pairs. In this example, S-D pairs 3-8 and 9 get benefits, whereas S-D pairs 1 and 4 suffer from performance degradation. This also explains why even with a small target rate as low as $5 \mathrm{~kb} / \mathrm{s}$, the outage probability is not small, as shown in Fig. 5. However, we can handle these fairness issues by incorporating other schemes in our selection framework. One can employ proportional fairness such as coefficients [19] 


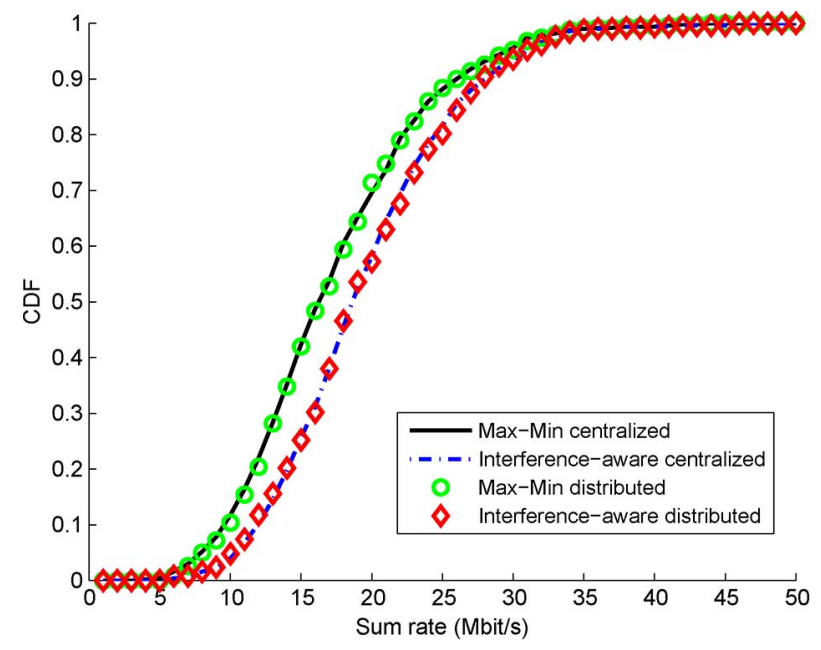

Fig. 8. Comparison between centralized and distributed relay selection schemes. We can see that the distributed relay selection scheme has similar performance as the centralized relay selection scheme.

or incorporate the queue-length information into the proposed selection metric. For example, higher priority is given to the S-D pairs with longer queues to use a better relay so that the interference it causes to other transmissions is less penalized. The detailed scheme design is left for our future work.

Fig. 8 shows the performance comparison between distributed (based on the DAP algorithm) and centralized relay selection algorithms (based on the Hungarian algorithm), for both conventional and interference-aware metrics. It is proven that the distributed relay selection scheme performs similarly to the centralized relay selection scheme. Therefore, our method can be well utilized in distributed scenarios, e.g., wireless ad hoc networks.

\section{CONCLUSION}

In this paper, we have proposed an interference-aware relay selection scheme for cooperative networks with concurrent relay-assisted transmissions. We model the relay selection as a weighted bipartite matching problem, where the S-D pairs and the relays are the two sides, and the weights are set according to the proposed interference-aware relay selection metric. To get this metric, the impact of interference from concurrent relay transmissions on a particular relay-to-destination link is decoupled based on the lower and upper bounds of the link rates. The relay selection metric is a linear combination of $\log$ functions of relay-to-destination SNRs. The first part of the metric represents the expected two-hop interference-free rate for the $\mathrm{S}-\mathrm{D}$ pair by selecting a relay, and the second part represents the rate lost of others due to the interference from the relay. A distributed relay selection algorithm is proposed based on matching game theory, and its performance is very close to the centralized one based on the Hungarian algorithm. Our scheme improves the sum rate by over $15 \%$ compared with the conventional max-min relay selection scheme. Since the objective is to maximize sum rate, the proposed scheme is not rate-fair for different $S-D$ pairs, and guaranteeing fairness is left for our future investigation.

\section{ACKNOWLEDGMENT}

The authors would like to thank D. Cao and Z. Yang for helpful discussions.

\section{REFERENCES}

[1] J. Laneman and G. Wornell, "Distributed space-time-coded protocols for exploiting cooperative diversity in wireless networks," IEEE Trans. Inf. Theory, vol. 49, no. 10, pp. 2415-2425, Oct. 2003.

[2] J. Laneman, D. Tse, and G. Wornell, "Cooperative diversity in wireless networks: Efficient protocols and outage behavior," IEEE Trans. Inf. Theory, vol. 50, no. 12, pp. 3062-3080, Dec. 2004.

[3] Y. Yang, H. Hu, J. Xu, and G. Mao, "Relay technologies for WiMax and LTE-advanced mobile systems," IEEE Commun. Mag., vol. 47, no. 10, pp. 100-105, Oct. 2009.

[4] Y. Zhu and H. Zheng, "Understanding the impact of interference on collaborative relays," IEEE Trans. Mobile Comput., vol. 7, no. 6, pp. 724 736, Jun. 2008

[5] J. Xu, S. Zhou, and Z. Niu, "Interference-aware relay selection for multiple source-destination cooperative networks," in Proc. APCC, Oct. 2009, pp. 338-341.

[6] S. Zhou, H. Xiao, and Z. Niu, "Distributed power control for interferencelimited cooperative relay networks," in Proc. IEEE ICC, Jun. 2009, pp. 1-6.

[7] A. Host-Madsen and J. Zhang, "Capacity bounds and power allocation for wireless relay channels," IEEE Trans. Inf. Theory, vol. 51, no. 6, pp. 2020-2040, Jun. 2005.

[8] A. Bletsas, A. Khisti, D. P. Reed, and A. Lippman, "A simple cooperative diversity method based on network path selection," IEEE J. Sel. Areas Commun., vol. 24, no. 3, pp. 659-672, Mar. 2006.

[9] A. S. Ibrahim, A. K. Sadek, W. Su, and K. J. R. Liu, "Relay selection in multi-node cooperative communications: When to cooperate and whom to cooperate with?" in Proc. IEEE GLOBECOM, Nov. 2006, pp. 1-5.

[10] A. K. Sadek, Z. Han, and J. R. Liu, "A distributed relay-assignment algorithm for cooperative communication in wireless networks," in Proc. IEEE ICC, Jun. 2006, pp. 1592-1597.

[11] B. Wang, Z. Han, and K. J. Ray Liu, "Distributed relay selection and power control for multiuser cooperative communication networks using Stackelberg game," IEEE Trans. Mobile Comput., vol. 8, no. 7, pp. 975990, Jul. 2009.

[12] Z. Han and H. V. Poor, "Coalition games with cooperative transmission: A cure for the curse of boundary nodes in selfish packet-forwarding wireless networks," IEEE Trans. Commun., vol. 57, no. 1, pp. 203-213, Jan. 2009.

[13] N. Shastry and R. S. Adve, "Stimulating cooperative diversity in wireless ad hoc networks through pricing," in Proc. IEEE ICC, Jun. 2006, pp. 3747-3752.

[14] D. Li, J. Liu, Y. Xu, X. Wang, and W. Chen, "Distributed relay-source matching for cooperative wireless networks using two-sided market games," in Proc. IEEE GLOBECOM, Dec. 2009, pp. 1-5.

[15] I. Krikidis, J. Thompson, S. McLaughlin, and N. Goertz, "Max-min relay selection for legacy amplify-and-forward systems with interference," IEEE Trans. Wireless Commun., vol. 8, no. 6, pp. 3016-3027, Jun. 2009.

[16] J. Si, Z. Li, and Z. Liu, "Outage probability of opportunistic relaying in rayleigh fading channels with multiple interferers," IEEE Signal Process. Lett., vol. 17, no. 5, pp. 445-448, May 2010.

[17] J. Huang, R. Barry, and M. Honig, "Distributed interference compensation for wireless networks," IEEE J. Sel. Areas Commun., vol. 24, no. 5, pp. 1074-1084, May 2006.

[18] Z. Guan, T. Melodia, D. Yuan, and D. A. Pados, "Distributed spectrum management and relay selection in interference-limited cooperative wireless networks," in Proc. ACM MobiCom, New York, NY, USA, Sep. 2011, pp. 229-240.

[19] M. Kountouris and D. Gesbert, "Memory-based opportunistic multiuser beamforming," in Proc. IEEE ISIT, Sep. 2005, pp. 1426-1430.

[20] P. Laureti and Y. C. Zhang, "Matching games with partial information," in Physica A: Statistical Machanics and its Applications. Amsterdam, The Netherlands: Elsevier, 2003.

[21] T. S. Rappaport, Wireless Communications: Principles and Practice. Englewood Cliffs, NJ, USA: Prentice-Hall, 1996.

[22] D. B. West, Introduction to Graph Theory, 2nd ed. Englewood Cliffs, NJ, USA: Prentice-Hall, 2000.

[23] D. Gusfield and R. W. Irving, The Stable Marriage Problem: Structure and Algorithms. Cambridge, MA, USA: MIT Press, 1989. 


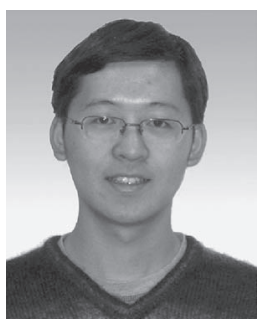

Sheng Zhou (S'06-M'12) received the B.S. and $\mathrm{Ph} . \mathrm{D}$. degrees in electronic engineering from Tsinghua University, Beijing, China, in 2005 and 2011, respectively.

From January to June 2010, he was a Visiting Student with Wireless System Laboratory, Department of Electrical Engineering, Stanford University, Stanford, CA, USA. He is currently a Postdoctoral Scholar with the Department of Electronic Engineering, Tsinghua University. His research interests include cross-layer design for multiple-antenna systems, cooperative transmission in cellular systems, and green wireless cellular communications.

Dr. Zhou co-received the Best Paper Award from the 15th Asia-Pacific Conference on Communications and from the 23th IEEE International Conference on Communication Technology in 2009 and 2011, respectively.

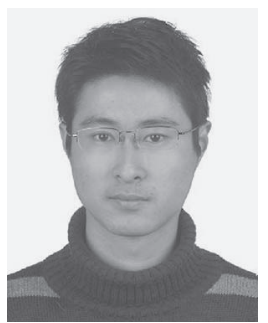

Jie Xu (S'09) received the B.S. and M.S. degrees in electronic engineering from Tsinghua University, Beijing, China, in 2008 and 2010, respectively. He is currently working toward the Ph.D. degree with the Department of Electrical Engineering, University of California, Los Angeles, USA

His main research interests include game theory and strategic design in multiagent networks with applications to cooperative communications, online markets, and smart grids.

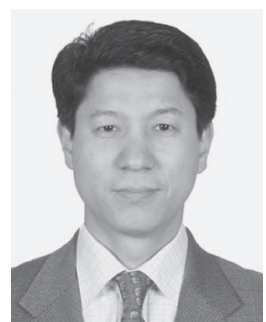

Zhisheng Niu (M'98-SM'99-F'12) received the B.S. degree from Beijing Jiaotong University, Beijing, China, in 1985 and the M.E. and D.E. degrees from Toyohashi University of Technology, Toyohashi, Japan, in 1989 and 1992, respectively.

He spent two years with Fujitsu Laboratories Ltd., Kawasaki, Japan. Since 1994, he has been with Tsinghua University, Beijing, China, where he is currently a Professor with the Department of Electronic Engineering and the Deputy Dean with the School of Information Science and Technology. His main research interests include queueing theory, traffic engineering, mobile Internet, radio resource management of wireless networks, and green communication and networks.

Dr. Niu was a council member of the Chinese Institute of Electronics from 2006 to 2010, the Vice-Chair of the Information and Communication Network Committee of the Chinese Institute of Communication from 2008 to 2012, a Councilor of The Institute of Electronics, Information, and Communication Engineers (IEICE) of Japan from 2009 to 2011, and the Membership Development Coordinator of IEEE Region 10 from 2009 to 2010. In particular, with the IEEE Communication Society (IEEE ComSoc), he has served as the Chair of the IEEE ComSoc Beijing Chapter from 2001 to 2008, the Director of the IEEE ComSoc Asia-Pacific Region from 2008 to 2009, the Editor for the IEEE Wireless Communication MaGaZiNe from 2009 to 2012, the Director of IEEE ComSoc Conference Publications from 2010 to 2011, and a member of the Award (2011-2013), Emerging Technologies (2010-2012), Online Content (2010-2012), and Strategy Planning Committees. He also served as the General Cochair of the 15th Asia-Pacific Conference on Communications (APCC) and the Fifth International Conference on Wireless Communications, Networking, and Mobile Computing in 2009; the Technical Program Committee Cochair of APCC in 2004, the International Conference on Communications in 2008, the 19th Annual Wireless and Optical Communications Conference in 2010, and the First IEEE International Conference on Communications in China; the Panel Cochair of the IEEE Wireless Communications and Wireless Conference in 2010; the Tutorial Cochair of the 72nd IEEE Vehicular Technology Conference in 2010 and the IEEE Global Communications Conference in 2012; and the Publicity Cochair of the 21st Annual IEEE International Symposium on Personal, Indoor, and Mobile Radio Communications in 2010 and the Wireless Communications and Networking Conference in 2002. He is currently a Fellow of IEICE and a distinguished Lecturer of IEEE ComSoc. He was also a Guest Coeditor for the IEEE WIRELESS COMMUNICATION MAGAZINE SPeCial Issue on Green Radio Communications and Networks (published on October 2011) and the Communication Networks Special Issue on Green Communication Networks (published in 2012). He received Best Paper Awards from the 13th and 15th APCC in 2007 and 2009, respectively, and the Outstanding Young Researcher Award from the Natural Science Foundation of China in 2009. 